Z. klin. Chem. u. klin. Biochem.

8. Jg., S. 208-211, Mai 1970

\title{
Formation of Zinc Chelates from Porphyrin Methyl Esters for Spectrophotometric Analysis')
}

\author{
By M. Doss \\ From the Clinical Biocbemistry Laboratory, Hygiene Institute of the University Marburg/Labn
}

(Eingegangen am 9. Februar 1970)

A specific and sensitive method is described for the spectrophotometric analysis of porphytin methyl esters in the form of their zine chelates.

Es wird eine spezifische und empfindliche Methode zur spektrophotometrischen Analyse der Porphyrine beschtieben, die in Form ihrer Methylester in Zinkchelate umgewandelt werden.

The quantitative analysis of porphyrins is carried out by means of spectrophotometry or fluorometry. Spectrophotometric determination offers an advantage in that extinction coefficients for the absorption maxima can be established under standard conditions which can be duplicated, in other laboratories; thus, comparable measurements are possible. The molat absorptivity and the stability of the porphyrins increase when a metal ion is incorporated into central position of the tetrapyrrole ring, forming metal complexes of the chelate type. Studies on copper, zinc, magnesium, nickel, and cadmium showed the absorption in the Soret band to be greatest for the zinc chelates. On the basis of this observation a sensitive spectrophotometric method has been developed for the analysis of porphyrins. As a prerequisite the porphyrins must be in the form of their methyl esters.

\section{Methods}

The analysis of porphyrins from biological material requires certain preparatory procedures. The methods developed in this laboratory for preparing the samples for spectrophotometric analysis are based on the following steps:

1. Esterification of the free porphyrin acids, e. g., from liver tissue $(1,2)$, urine $(2,3)$, stomach or duodenal secretions (4), blood cells $(5,6)$ or bacteria $(7)$, to their methyl esters.

2. Extraction of the porphyrin methyl esters using chloroform.

3. Thin-layer chromatography on silica gel film.

4. Elution of the porphyrin zones of the chromatogram.

This procedure has been presented in detail in a previous paper (8).

For the formation of zinc chelates, the solvent is evaporated from the porphyrin methyl ester solution, and the ester dissolved in a solution of zinc(II) acetate $0.5 \%(\mathrm{w} / \mathrm{v})$ in chloroform-methanol $(19+1, \nabla+\nabla)$. The conversion of porphyrin mixture containing up to $3 \mu \mathrm{g}$ of porphyrins with 8 to 2 carboxylic methyl ester groups in $1 \mathrm{~m} l$ of reagent is complete after $40 \mathrm{~min}$ at room temperature $\left(20^{\circ} \pm 2\right)$. The spectrum is then recorded between 600 and $380 \mathrm{~nm}$.

1) Presented in part at VIIth International Congress of Clinical Chemistry, Genève/Evian, September 8-13, 1969.

\section{Results}

Porphyrin methyl esters are transformed into zinc chelates, which exhibit a substantially higher molar absorptivity than do the pure, metal-free porphyrin methyl esters in chloroform solution (9). In addition to the Soret band, the absorption spectrum of porphyrin methyl esters dissolved in chloroform, ether, or dioxan possesses four secondary maxima (I, II, III and IV (10)), which lie between 650 and $350 \mathrm{~nm}$. The spectrum of the metal chelates of the porphyrins, however, exhibits only two relatively small absorption bands within the visible range $(\alpha$ and $\beta$ ) (Fig. 1, Tab. 1).

The absorption maxima of the zinc chelates of proto-, copro- and uroporphyrin methyl esters in the spectrum between 600 and $380 \mathrm{~nm}$ are presented along with the millimolar extinction coefficients in the Soret band in Table 1.

Tab. 1

Absorption maxima and millimolar extinction coefficients $\left(\varepsilon_{\mathrm{mM}}\right)$ of $\mathrm{Zn}$ chelates of proto-, copro- and uroporphyrin methyl esters. Solution both for the formation and spectrophotometric measurement
of porphyrin ester $\mathrm{Zn}$ chelates: $\mathrm{Zn}$ acetate $0.5 \%(\mathrm{w} / \mathrm{v})$ in chloroformmethanol $(19+1, v+v)$

\begin{tabular}{lcccc}
\hline Porphyrin & $\begin{array}{c}\text { Soret } \\
(\mathrm{nm})\end{array}$ & $\begin{array}{c}\beta \\
(\mathrm{nm})\end{array}$ & $\begin{array}{c}\alpha \\
(\mathrm{nm})\end{array}$ & $\begin{array}{c}\text { Soret } \\
\boldsymbol{\varepsilon}_{\mathrm{mM}}\end{array}$ \\
\hline Proto- & 418 & 546 & 583 & 267 \\
Copro- & 410 & 540 & 575 & 353 \\
Uro- & 415 & 544 & 579 & 406 \\
\hline
\end{tabular}

The velocity of reaction is a function of the number of carboxyl groups in each molecule (Fig. 2), as well as of the concentration of the metal in the solution (Fig. 3). The curves in figure 3 show the kinetics of the conversion of proto- and uroporphyrin methyl esters in zinc acetate solution of various concentrations. For the routine transformation and analysis of porphyrin esters, a $0.5 \%$ (w/v) zinc acetate solution in chloroform-methanol $(19+1, v+v)$ was chosen. The reverse transformation of the porphyrin methyl ester $\mathrm{Zn}$ chelates into the metal-free substances can be achieved by adding methanol-sulphuric acid $(95+5, v+v)$, in analogy to the esterification procedures for free porphyrins. The 


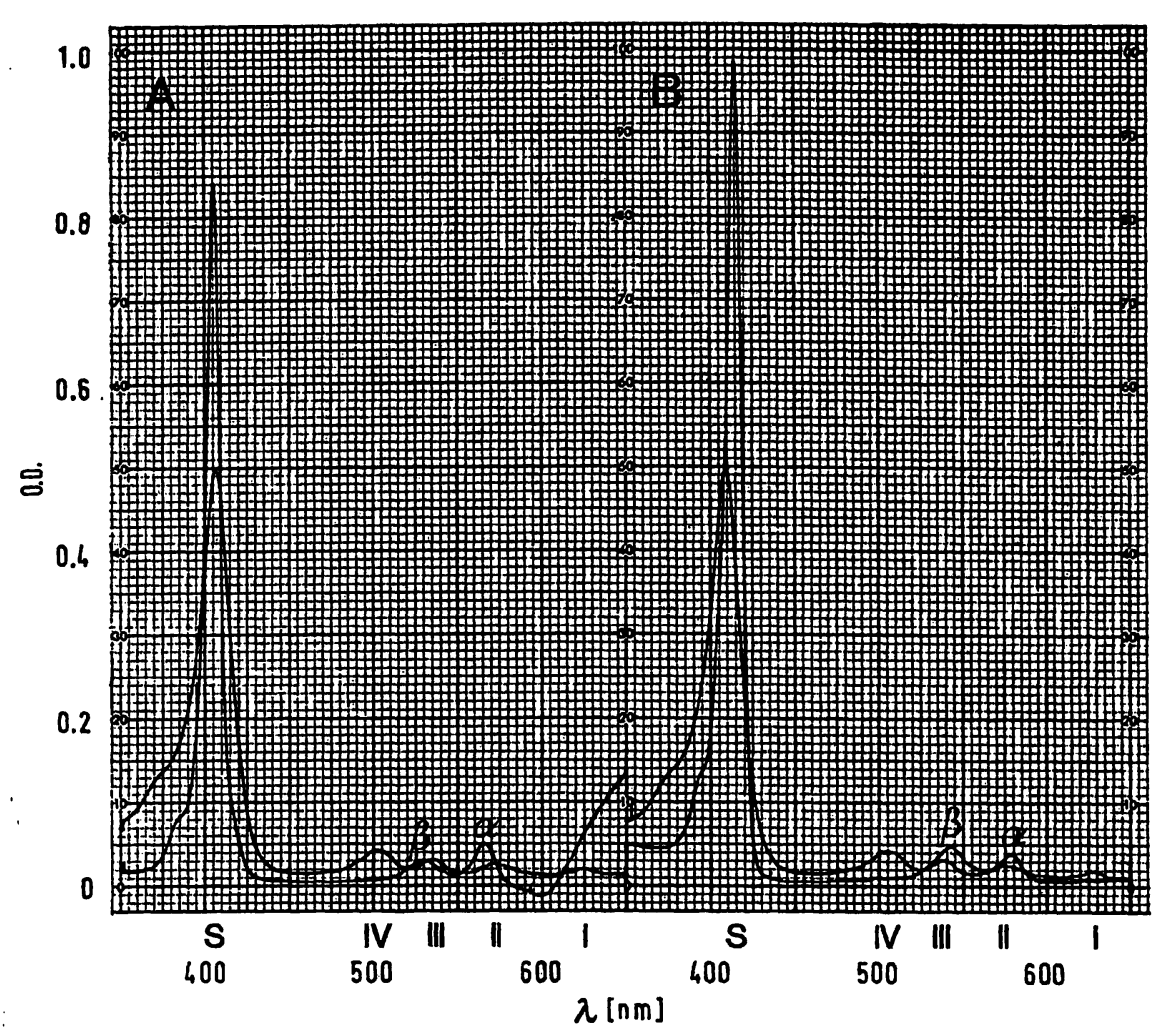

Fig. 1

Absorption spectra of uroporphyrin III octamethyl ester $(2.31 \mu \mathrm{M}, \mathrm{d}=1 \mathrm{~cm})$ in chloroform (O.D. $=0.5, A$ and $B$ ), as $C u$ chelate (A) and as $\mathrm{Zn}$ chelate (B). The copper complex is formed following evaporation of the solvent by dissolving the uroporphyrin methyl ester in a solution of $\mathrm{Cu}(\mathrm{II})$-acetate $0.1 \%(\mathrm{w} / \mathrm{v})$ in chloroform-methanol $(19+1, v+v)$. Likewise, following complete removal of the chloroform, the uroporphyrin methyl ester was dissolved in the same volume of a solution of $\mathrm{Zn}$ acetate $0.5 \%(w / v)$ in chloroform-methanol $(19+1$, $v+v$ ) for conversion into zinc chelates. $S=$ Soret maximum; I-IV = secondary maxima of porphyrin ester in chloroform solution; $\alpha$ and $\beta=$ secondary maxima of chelates. The spectra were recorded between 650 and 350 $\mathrm{nm}$. Spectrophotometer Beckman DB-G with ten-inch potentiometric recorder

Fig. 2

The kinetics of zinc chelate formation from porphyrin methyl esters as a function of the number of methyl ester groups in each molecule. $\mathrm{P}=$ Proto, $\mathrm{C}=$ Copro, $\mathrm{U}=$ Uro

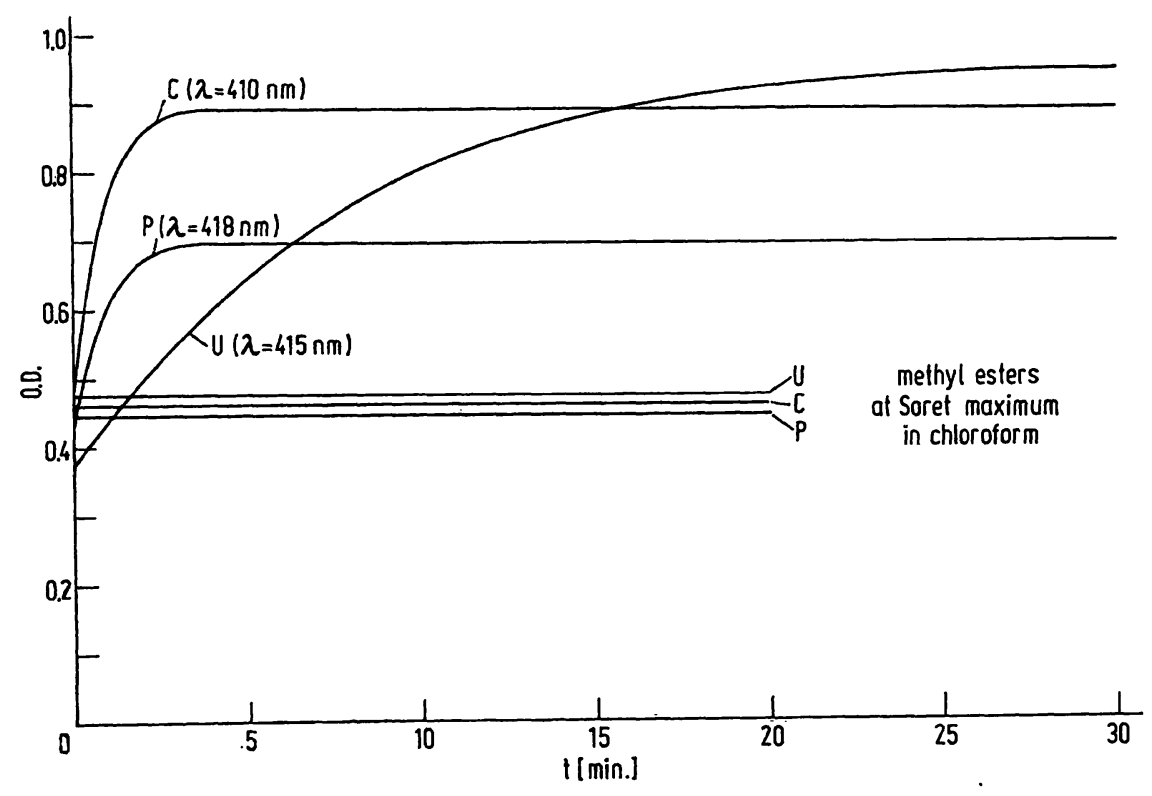

extinction coefficients assembled in Table 1 reveal the optimal range of measurement on a non-expanded scale to lie between 0.03 and $1 \mu \mathrm{g}$ of a porphyrin methyl ester, provided that the volume measured is $1 \mathrm{ml}$ and the path length $2 \mathrm{~cm}$. These conditions are sufficient for the spectrophotometric determination of the porphyrins from $5 \mathrm{ml}$ of normal urine, following the separation of their methyl esters (8) in thin-layer chromatography.

Zinc chelates of porphyrin methyl esters exhibit an orange fluorescence under long-wave UV light (366 nm). This enables us to observe the formation of zinc chelates in the solution with the naked eye. A transition from the intense red fluorescence of the porphyrins into a paler orange fluorescence characterizes the completion of the reaction. On the basis of the same color difference, porphyrin zinc chelates on adsorbent layers (8) can be easily and unambiguously distinguished and recognized. By identical molat concentrations of the porphyrins the visible fluorescence of the zinc chelates is weaker than that of the metal-free substances. The fluorescence excitation and emission maxima of proto-, copro- and uroporphyrin methyl esters and the corresponding zinc chelates are presented in Table 2.

The separation of the zinc chelates by means of thinlayer chromatography is carried out similarly to that of the metal-free porphyrin esters using the solvent system benzene-ethyl acetate-methanol $(85+13.5+1.5, \mathrm{v}+$ v) (5). As shown in figure 4 and 5 , the behavior of the zinc chelates is characterized by somewhat lower $R_{F}$ values than those of the corresponding metal-free porphyrin esters. For the chromatogram in figure 4, a mixture of reference substances consisting of proto-, 

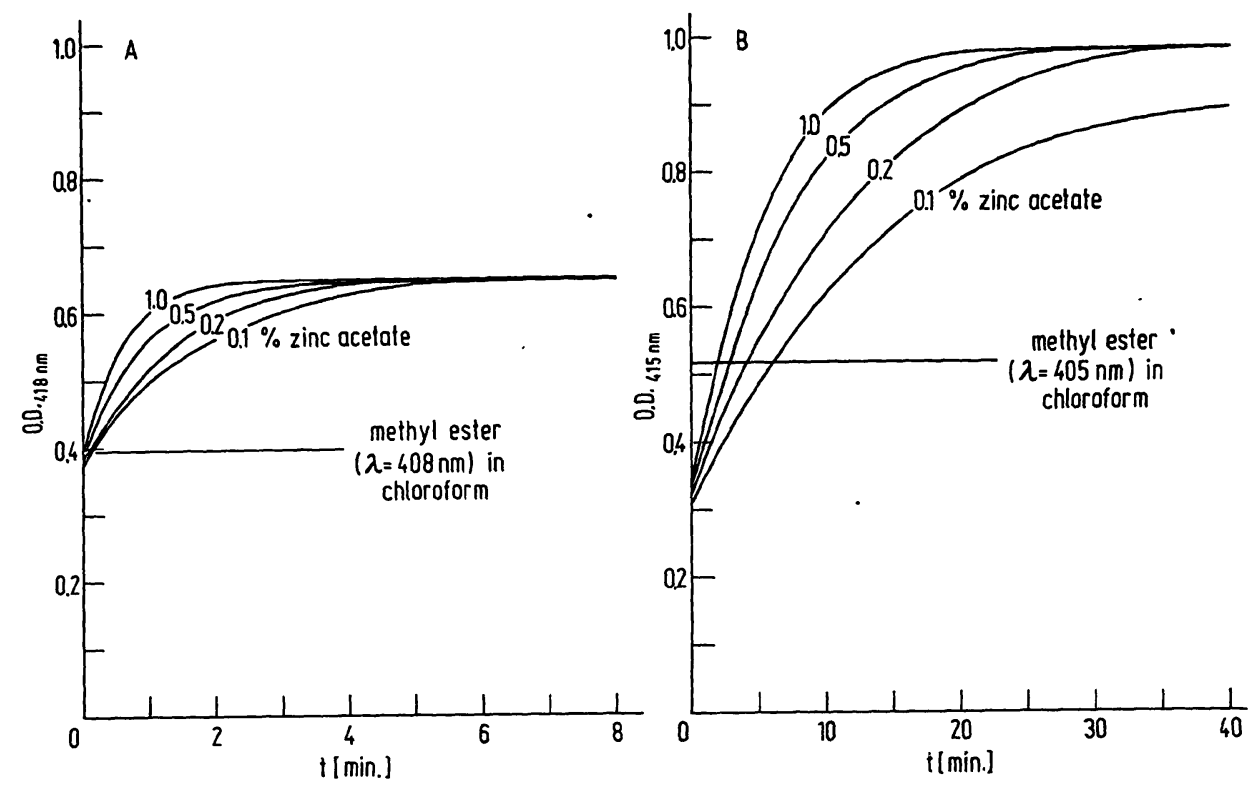

Fig. 3

Kinetics of zinc chelate formation from protoporphyrin IX (A) and uroporphyrin III (B) methyl esters in response to the concentration of the metal (zinc acetate: $0.1,0.2,0.5$ and $1.0 \%, \mathrm{w} / \mathrm{v})$ in the reagent solution

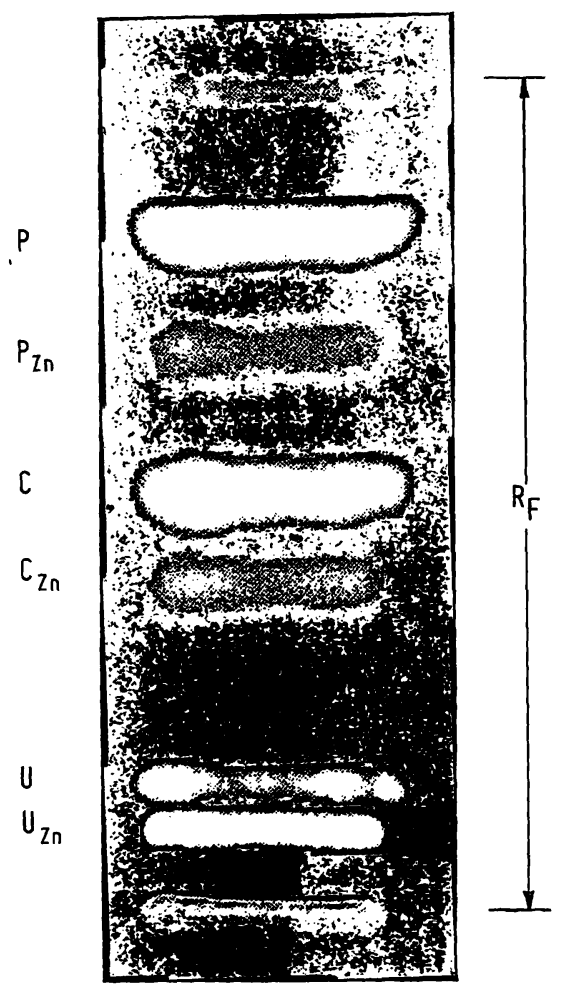

Fig. 4

Comparison between the separation of uro- (U), copro- (C), and proto(P)-porphyrin methyl esters and the same porphyrin methyl esters as zinc chelates $\left(U_{Z_{n}}, C_{Z_{n}}, P_{Z_{n}}\right.$ ) on a DC card SI (Riedel-de Haën $37361)$ in the benzene-ethyl acetate-methanol system $(85+13.5+$ $15 \mathrm{~V}+\mathrm{v})$. Porphyrin methyl ester $\mathrm{Zn}$ complexes in solution and in the chromatoram exhibit orange fluorescencexes in solution and in violet radiation as compared to the clear red fluorescence of pure porphyrin carboxylic acids or esters

copro- and uroporphyrin methyl esters was converted to zinc chelates, which were then separated next to the original substances. This experiment suggests the possibility of converting the porphyrin methyl esters of a biological extract into chelates before separating them in thin-layer chromatography, i. e., to separate the zinc chelates, such has been done in the chromatogram in figure 5 . Before the application of the porphyrin methyl ester zinc chelates to the silica gel layer, and after complete chelate formation, the solvent is evapo-

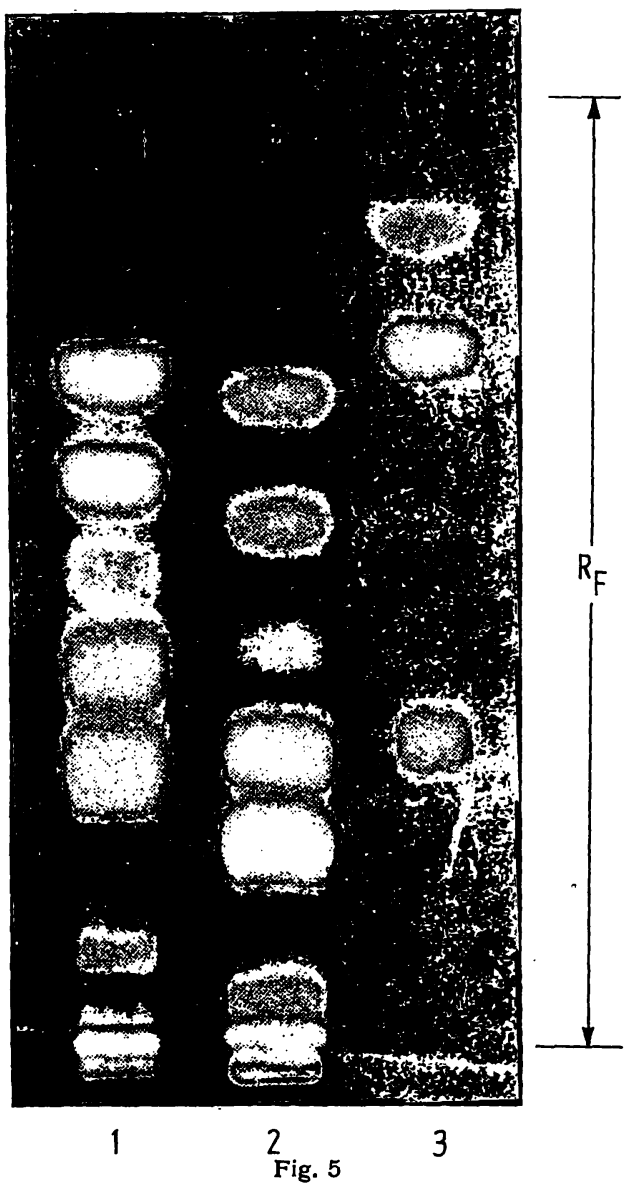

Comparison between the separation of porphyrin methyl esters (1) and the porphyrin methyl esters zinc chelates (2) on a Silica Gel $F_{254}$ plate $(10 \times 20 \mathrm{~cm}$, Merck 5729) after two-fold development in the benzene-ethyl acetate-methanol system $(85+13.5+1.5, \mathrm{v}+\mathrm{v})$. The reference substances, uro-, copro- and protoporphyrin methyl esters, are separated in position 3. Porphyrin methyl esters for position 1 and 2 were prepared from $10 \mathrm{ml}$ each of urine from a patient with
porphyria cutanea tarda. Sample 1 was applied without further treatment; sample 2 was converted to $\mathrm{Zn}$ chelates

rated from the zinc acetate reagent. Then the chelates are redissolved in pure chloroform for application. According to the source of porphyrins, the chromatogram is developed either in the above-mentioned solvent system or stepwise (8). For spectrophotometric analysis, the porphyrin methyl ester zinc chelates are eluted from the 
Tab. 2

Fluorescence excitation and emission spectra*)

\begin{tabular}{lccc}
\hline $\begin{array}{c}\text { Porphyrin } \\
\text { methyl esters }\end{array}$ & $\begin{array}{c}\text { Absorption } \\
\text { maxima(Soret) } \\
( \pm 0.5 \mathrm{~nm})\end{array}$ & $\begin{array}{c}\text { Fluorescence } \\
\text { excitation } \\
\text { maxima } \\
( \pm 2 \mathrm{~nm})\end{array}$ & $\begin{array}{c}\text { Fluorescence } \\
\text { emission } \\
\text { maxima } \\
( \pm 1 \mathrm{~nm})\end{array}$ \\
\hline Proto- & 407.5 & 410 & 632 \\
Copro- & 399.5 & 401 & 623 \\
Uro- & 405.5 & 405 & 627 \\
Proto-Zn & 418 & 417 & 588 \\
Copro-Zn & 410 & 409 & 578 \\
Uro-Zn & 415 & 414 & 582 \\
\hline
\end{tabular}

*) Measured in chloroform solution (containing $5 \%$ methanol for $\mathrm{Zn}$ chelates) on a Hitachi Perkin-Elmer Fluorescence Spectrophotometer MPF-2A.

silica gel with chloroform-methanol $(19+1, v+v)$, and then either immediately measured, or in chloroform p. a. With chloroform-methanol $(19+1, v+v)$ the zinc chelates are completely eluted even without filtration, which has been prescribed for porphyrin methyl ester (9).

Figure 5 shows the porphyrin mixture from a patient with Porphyria cutanea tarda. On track 1 , the porphyrin methyl ester extracted from $10 \mathrm{~m} l$ of urine is separated; the porphyrin esters of the parallel sample were converted to zinc chelates before being applied to the thinlayer plate. The porphyrin methyl esters and their zinc



Scanning Direction $\longrightarrow$.

Fig. 6

Fluorescence scan of proto-, copro- and uroporphyrin $(P, C, U)$ as methyl esters (A) and as methyl ester $\mathrm{Zn}$ chelates (B). Both were separated on a Riedel-de Haên DC card SI and
Camag-Turner TLC-Scanner (8) chelates separate to form a pattern analogous to that shown in figure 4 , whereby the respective zinc chelates migrate a shorter distance. Porphyrin $\mathrm{Zn}$ chelates in a chromatogram can be fluorometrically recorded on the Camag-Turner TLC-Scanner (Fig. 6), with which the direct fluorescence analysis of the porphyrins on chromatograms is carried out (8).

\section{Discussion}

The formation of zinc chelates from porphyrin methyl esters represents a new method for the spectrophotometric and fluorometric analysis and identification, chromatographic separation, and qualitative demonstration of porphyrins. Among these possibilities, only the spectrophotometric analysis of porphyrins in the form of zinc chelates is of practical significance for use in clinical chemistry. In order that the chelates may be formed under the conditions described, it is necessary that the porphyrins be present as methyl esters. The analysis and technical advantages of working with porphyrins in the form of their methyl esters have been discussed in a preceding work (8).

The formation of zinc chelates from meso- and protoporphyrin occurs more rapidly than from other porphyrins. In agreement with the values cited by FALK (10), we found that electronegative substituents exert a negative influence on the rate of formation of metalloporphyrins. As shown in figure 2, the kinetics of zinc chelate formation from porphyrins changes as the number of carboxylic acid side chains increases.

The analysis of porphyrin methyl esters separated by TLC in form of their zinc chelates is especially appropriate for porphyrin bands containing less than $0.2 \mu \mathrm{g}$. An optimal estimate of the concentration in the chromatogram, based on the fluorescence, is sufficient for deciding which kind of quantitative analysis is to be used; higher concentrations are best measured in chloroform (9).

The advantages of spectrophotometric measurements of porphyrin methyl ester zinc chelates in comparison to copper chelates (11) are: 1 , pure chloroform can be used as a blank, and 2, zinc chelates fluoresce under long-wave UV light, whereas the copper chelates exhibit no fluorescence whatsoever under the same conditions.

This work was carried out with funds from the Deutsche Forschungsgemeinschaft, which I should like to thank for its generous support.

\section{References}

1. Doss, M., Klin. Wschr. 47, 1280 (1969). - 2. Doss, M., Dtsch. med. Wschr. 95, 959 (1970). - 3. Doss, M. and W.-K. PhrLIpp, this journal 7, 148 (1969). - 4. Doss, M. and L. FrLIPpINI, this journal 7, 306 (1969). - 5. Doss, M., Dtsch. med. Wschr. 93, 2223 (1968). - 6. Doss, M. and U. BoDE, this journal 6, 383
(1968). - 7. Doss, M., Biochim. biophysica Acta Amsterdam 170, 461 (1968). - 8. Doss, M., this journal 8, 197 (1970). - 9. Doss, M., Hoppe-Seyler's Z. physiol. Chem. 350, 499 (1969). - 10. FalK, J. E., Porphyrins and Metalloporphyrins, Elsevier, Amsterdam (1964). - 11. Doss, M., this journal 6, 498 (1968). 